\title{
Dielectric elastomer actuator for the measurement of cell traction forces with sub-cellular resolution
}

$\underline{\text { Samuel Rosset; Alexandre Poulin; Alicia Zollinger; Michael Smith; Herbert Shea }}$

Proc. SPIE 10163, Electroactive Polymer Actuators and Devices (EAPAD) 2017, 101630P (April 17, 2017); doi:10.1117/12.2258731

Copyright 2017 Society of Photo-Optical Instrumentation Engineers. One print or electronic copy may be made for personal use only. Systematic electronic or print reproduction and distribution, duplication of any material in this paper for a fee or for commercial purposes, or modification of the content of the paper are prohibited. 


\title{
Dielectric elastomer actuator for the measurement of cell traction forces with sub-cellular resolution
}

\author{
Samuel Rosset ${ }^{a}$, Alexandre Poulin ${ }^{a}$, Alicia Zollinger ${ }^{b}$, Michael Smith $^{b}$, and Herbert Shea ${ }^{a}$ \\ ${ }^{a}$ Ecole polytechnique fédérale de Lausanne (EPFL), Lausane, Switzerland \\ ${ }^{b}$ Boston University, Biomedical Engineering Department, Boston, USA.
}

\begin{abstract}
We report on the use of dielectric elastomer actuators (DEAs) to measure the traction force field of cells with subcellular resolution. The study of cellular electrochemical and mechanical response to deformation is an important area of research, as mechanotransduction has been shown to be linked with fundamental cell functions, or the progression of diseases such as cancer or atherosclerosis. Experimental cell mechanics is based on two fundamental concepts: the ability to measure cell stiffness, and to apply controlled strains to small clusters of cells. However, there is a lack of tools capable of applying precise deformation to a small cell population while being compatible with an inverted microscope (stable focal plane, transparency, compactness, etc.). Here, we use an anisotropically prestretched silicone-based DEA to deform a soft $(7.6 \mathrm{kPa})$ polyacrylamide gel on which the cells are cultured. An array of micro-dots of fluorescent fibronectin is transferred on the gel by micro-contact printing and serves as attachment points for the cells. In addition, the fluorescent dots (which have a diameter of $2 \mu \mathrm{m}$ with a spacing of $6 \mu \mathrm{m}$ ) are used during the experiment to monitor the traction forces of a single cell (or small cluster of cells). The cell locally exerts traction on the gel, thus deforming the matrix of dots. The position of dots versus time is monitored live when the cells are submitted to a uniaxial strain step. Our deformable bioreactor enables the measurement of the local stiffness of cells submitted to mechanical strain, and is fully compatible with an inverted microscope set-up.
\end{abstract}

Keywords: Dielectric elastomer actuators, fabrication process, manufacturability

\section{INTRODUCTION}

The measurement of cell contractility and the ability to apply controlled strains to cells are the two pillars of experimental cell mechanics and mechanobiology, which is in turn foundational for tissue engineering and regenerative medicine. ${ }^{1}$ However, there is a lack of integrated platforms capable of applying a controlled mechanical deformation with a high strain rate to small cell populations.

As an example of application in the field of mechanobiology, the stiffness and prestress of cells has been characterized by Canovic et al. using a technique called biomechanical imaging. ${ }^{2}$ It consists in culturing cells (in this case 3T3 fibroblasts) on a soft polyacrylamide gel. An array of microdots of fluorescent fibronectin is patterned on the soft gel and serves as attachment points for the cells (Figure $1 \mathrm{~A}$ ). Once the cells are attached, they exert traction on the gel, which deforms the array of fluorescent dots (Figure 1 B). From the generated strain field and mechanical properties of the gel, the traction forces of the cell can be calculated with a sub-cellular resolution (Figure $1 \mathrm{C}$ ). Cells reach a state of tensional homeostasis, at which intracellular and extracellular forces balance. When an external mechanical strain is applied to the cells, they respond by adjusting their mechanical response, which leads to the activation of signalling pathways, to the expression of genes, and to other mechanotransduction phenomena that characterise the response of cells to mechanical cues.

Although commercial devices are available to submit cells to mechanical deformation, such as Flexcell, they are usually not compatible with in-situ imaging systems. The measurement of cell contractility requires the cell culture to be located under a microscope during the experiment. In the biomechanical imaging of fibroblasts mentioned above, ${ }^{2}$ the cells are strained by indenting the gel between two parallel glass plates (Figure $1 \mathrm{D}$ ). However, indenting the gel to deform the cells cultured on its surface has several drawbacks: 1) The strain field

contact author: samuel.rosset@a3.epfl.ch

Electroactive Polymer Actuators and Devices (EAPAD) 2017, edited by Yoseph Bar-Cohen, Proc. of SPIE Vol. 10163, 101630P · C 2017 SPIE · CCC code: 0277-786X/17/\$18 · doi: 10.1117/12.2258731

Proc. of SPIE Vol. $10163101630 \mathrm{P}-1$ 


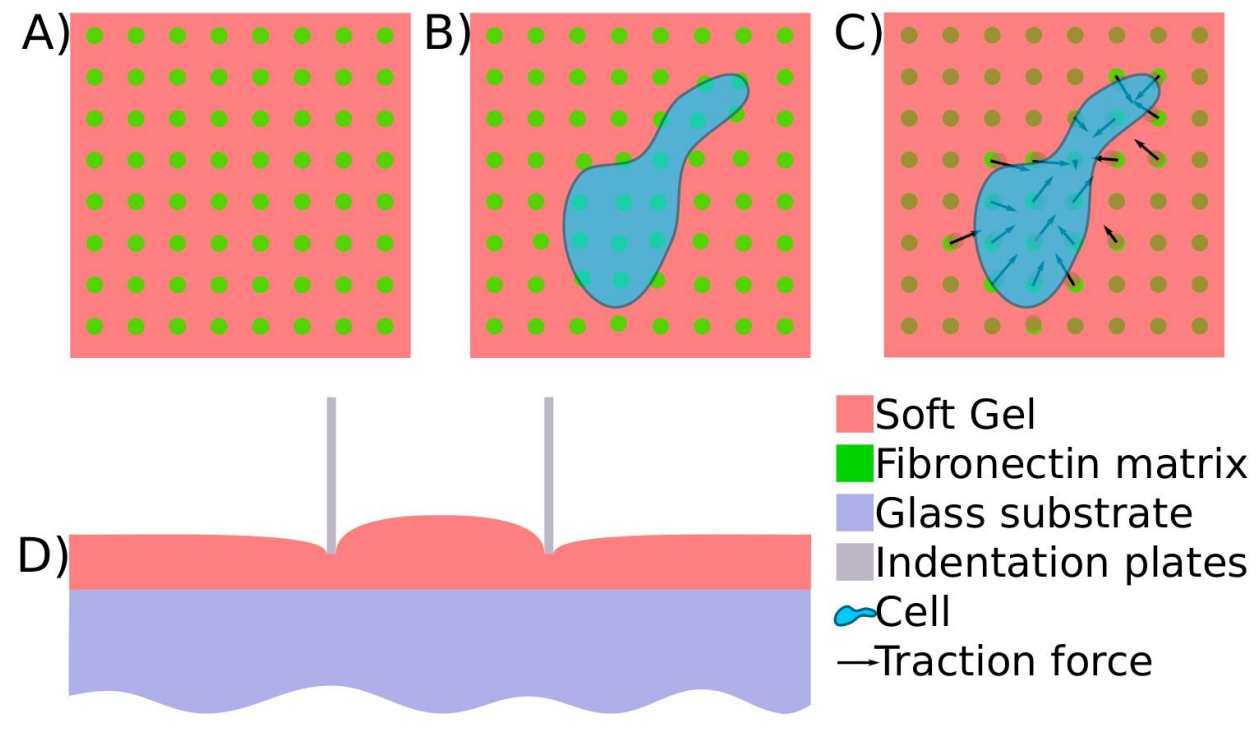

Figure 1. Principle of cell traction force measurement using biomechanical imaging: A) A matrix of fibronectin labelled with a fluorescent molecule is patterned on top of a soft polyacrylamide gel. B) Cells are cultured on the gel and attach to the fibronectin dots. They exert traction on the gel, which leads to a displacement of the dots. C) Fluorescence microscopy is used to take pictures of the matrix of dots at regular intervals. Image processing routines are used to compute the displacement of the dots, and therefore, the traction forces. D) To submit the cells to stretch, the gel is indented with two plates, leading to local deformation.

is inhomogeneous and difficult to control. 2) The in-plane strain is combined with an out-of-plane displacement. Because high-magnification objectives often have a very shallow depth of field, it is therefore difficult to keep the culture in focus. 3) It is difficult to build a setup that can indent the gel and fit under an inverted microscope, and 4) the amount of strain is difficult to control, and it is not trivial to impose high strain rates to the culture.

Here, we propose to use a planar dielectric elastomer actuator (DEA) to stretch the cells cultivated on the soft hydrogel. The use of DEA as a means to stretch the cells has many advantages compared to alternative methods, such as the plate indentation mentioned above. ${ }^{2}$ The electrostatic actuation enables an easy control of the applied strain, while keeping the cells in the same focal plane, thus making live imaging possible. The compact design also makes it possible to use the device directly under an inverted microscope.

We have recently presented how DEAs can be applied to mechanotransduction studies by investigating the effect of a periodic stretch on the orientation and shape of lymphatic endothelial cells (LECs). ${ }^{3}$ Briefly, a silicone membrane is anisotropically prestretched and fixed on a frame. Compliant electrodes made from a carbon black/silicone composite are patterned by pad-printing on both sides of the membrane. The electrodes are patterned to define a small $1.5 \mathrm{~mm} \times 0.5 \mathrm{~mm}$ zone without electrodes at the centre of the device, where the cells are stretched. The anisotropic prestretch ensures that the strain of the actuator occurs preferentially in one direction, thus providing unixial stretching of the population of cells located at the centre of the device. ${ }^{3-5}$ Cells are cultured on the complete device, but are only stretched at the centre, which makes it possible to compare the stretched population with a reference (i.e. unstretched) sample. The effect of the electric field on the cells has been studied, to show that the cells remain unaffected by the high electric field required to drive DEAs. ${ }^{3}$ This study demonstrates that DEAs are well adapted to mechanotransduction studies.

The actuator configuration used for this study is very similar to the one used for the LECs study mentioned above. However, because of the low seeding density required to have single cells (or clusters of a few cells) located on zones with a fibronectin dot matrix of good quality and contrast, the design was slightly modified to increase the stretched zones of the actuator, in order to increase the possible observation sites where a measurement can be made. Indeed, as explained in more details later (c.f. section 2), the fibronectin dot matrix, which is essential to measure the traction field of the cells is made with a manual micropatterning process which doesn't lead to a 


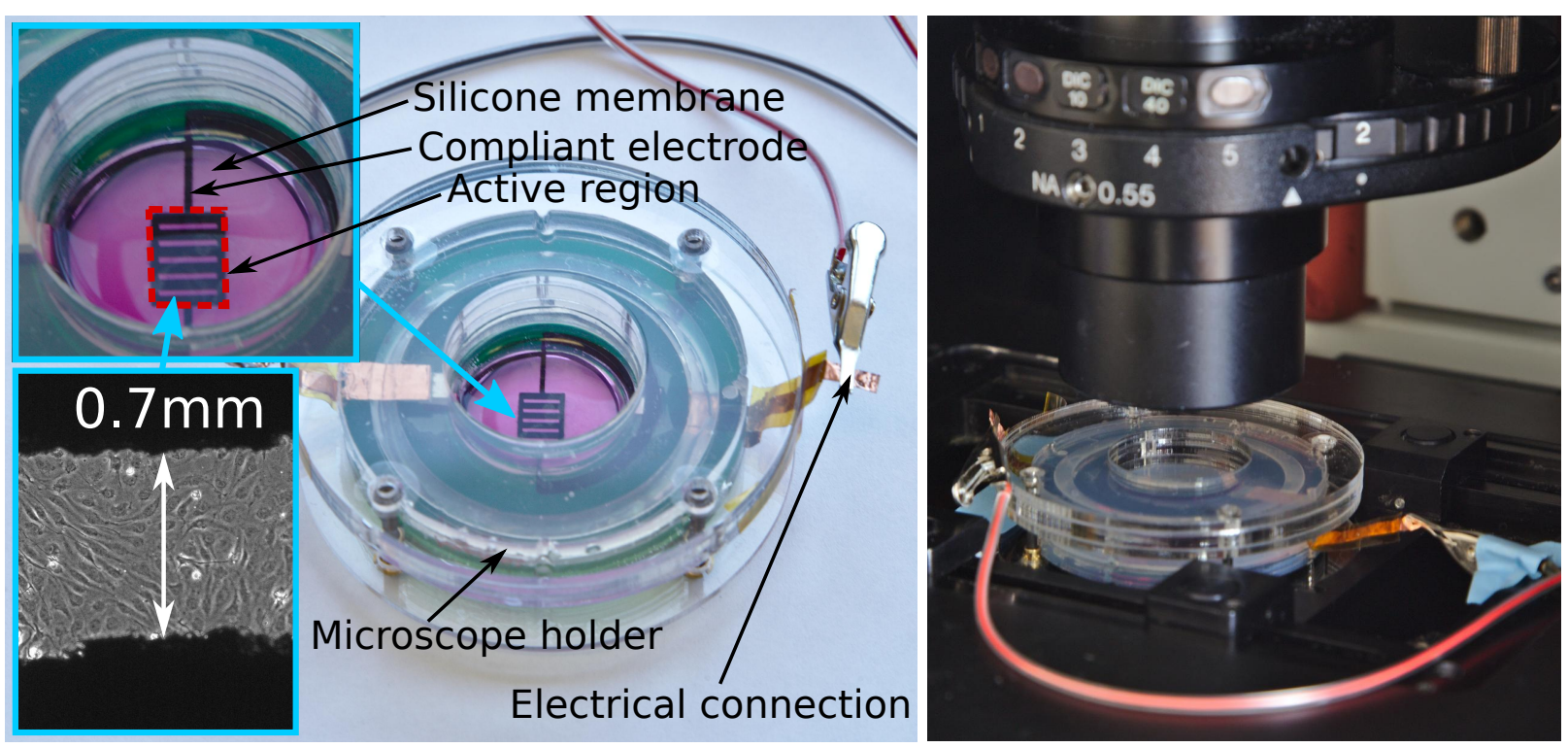

Figure 2. The actuator used for the study of cell traction forces consists of five $0.7 \mathrm{~mm} \times 4.5 \mathrm{~mm}$ transparent stretching zones surrounded by an electrode. Although cells are located on the whole device, only cells located in the 5 transparent zones are visible when the device is used with an inverted microscope. The device is placed into a holder that provides electrical connection and that can be loaded in an inverted microscope fitted with an incubation chamber.

good defect-free pattern over the whole surface of the gel. It is therefore necessary to maximize the area where cells can attach and be stretched, in order to increase the probability to have zones that combine a dot pattern which is useable by the image analysis routines and healthy single cells attached, in a zone that is submitted to strain. Consequently, we opted for an actuator containing five $0.7 \mathrm{~mm} \times 4.5 \mathrm{~mm}$ zones in which cells can be stretched and observed under a microscope (Figure 2). The device integrates a stretched membrane, patterned compliant electrodes that define the five stretching zones, and a cell culture chamber. It is mounted in a holder that provides electrical contacts to the device and that can be installed under an inverted microscope. For these experiments, the microscope included a whole incubating chamber around it, but DEA-based devices designed to stretch cells are compact enough to be mounted into portable incubators, such as what was done in the LEC study. ${ }^{3}$

\section{FABRICATION OF THE ACTUATOR}

A silicone Sylgard 186 membrane (Dow Corning) is cast on a PET foil coated with a sacrificial layer, using our standard methodology, ${ }^{6}$ and then anisotropically prestretched by a factor 2.7:1.2 and fixed on a frame with an inner diameter of $42 \mathrm{~mm}$. The electrodes are applied by pad printing using a steel cliché, ${ }^{6}$ and consists of a mixture of carbon black and silicone. After crosslinking of the electrodes, a $2 \mu \mathrm{m}$-thick passivation layer of soft silicone is applied by pad printing ${ }^{7}$ on the top side of the membrane to cover the electrodes, leaving part of the top electrode uncovered for the electrical access (Figure 3 left). Finally, a plastic ring with an inner diameter of $22 \mathrm{~mm}$ and a height of $5 \mathrm{~mm}$ is placed at the centre of the membrane and acts as a culture chamber. A metallic tape on one side of the ring provides electrical contact to the top electrode (Figure 3 right). Once the actuator part is finished (Figure $4 \mathrm{~A}$ ), it is tested to measure its strain and its ability to sustain electric field without breakdown.

The next steps consists in binding a thin $(30 \mu \mathrm{m}$ to $100 \mu \mathrm{m})$ layer of a soft $(7.6 \mathrm{kPa})$ polyacrylamide gel to the top surface of the actuator, and to transfer an array of fluorescent fibronectin dots on the surface of the gel. First, an array of microdots ( $2 \mu \mathrm{m}$ diameter and $6 \mu \mathrm{m}$ centre-to-centre separation) is patterned on a $20 \mathrm{~mm}$ diameter glass coverslip by micro-contact printing, using a soft silicone stamp (Figure $4 \mathrm{~B}$ ). The micro-patterning step is done manually, and some of the steps are critical, such as the time the solution is left to dry on the stamp, and most importantly the force applied on the stamp with the finger to transfer the pattern. Too little force 

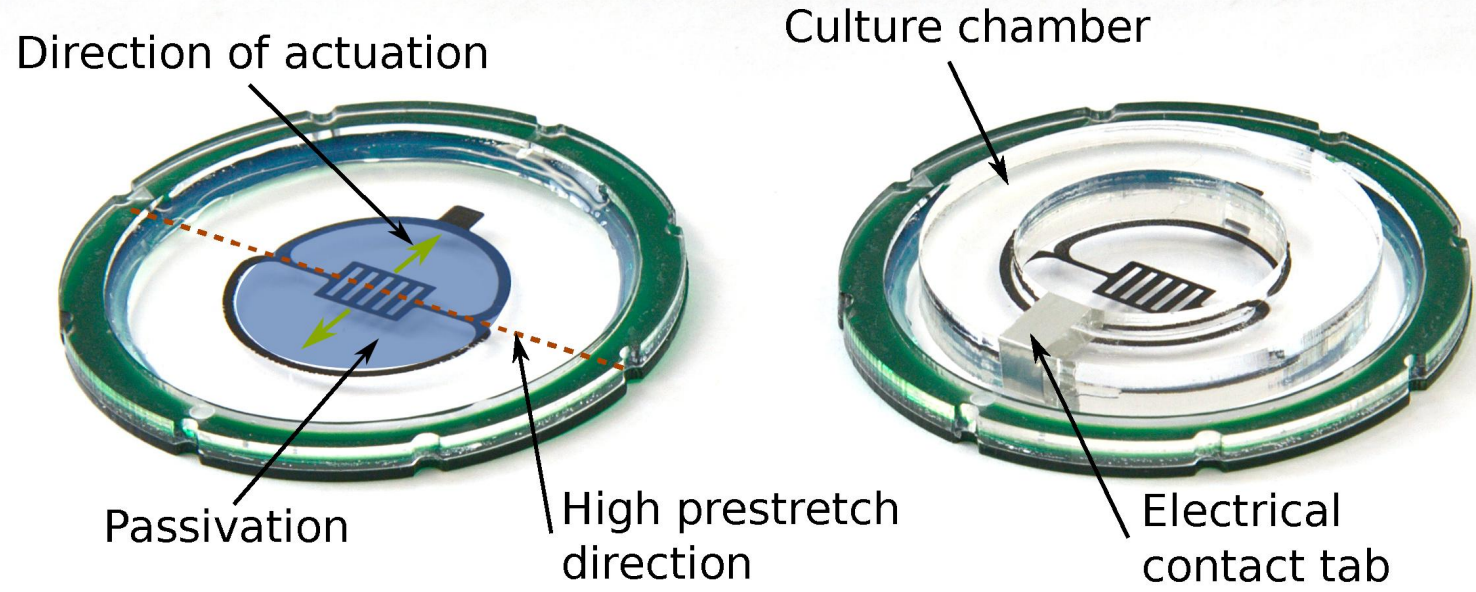

Figure 3. Left: actuator after application of the electrodes and passivation (in the shaded area). Right: actuator after application of the cell culture chamber with a metallic contact.
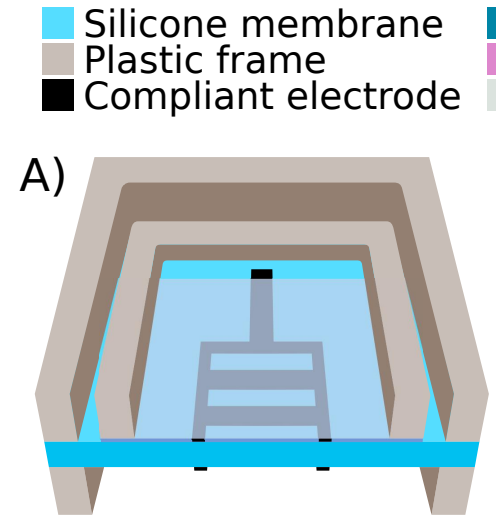

B)

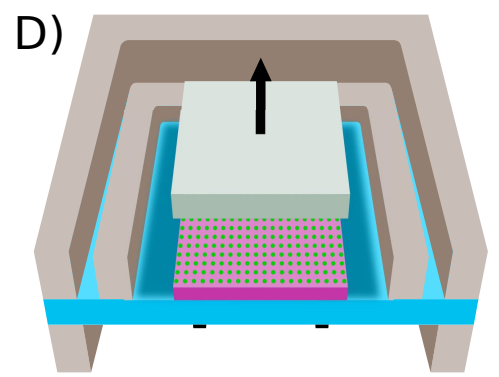

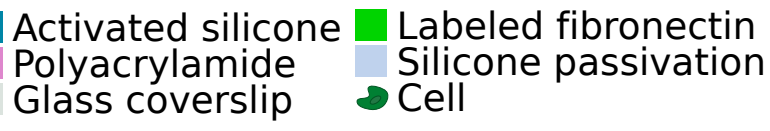
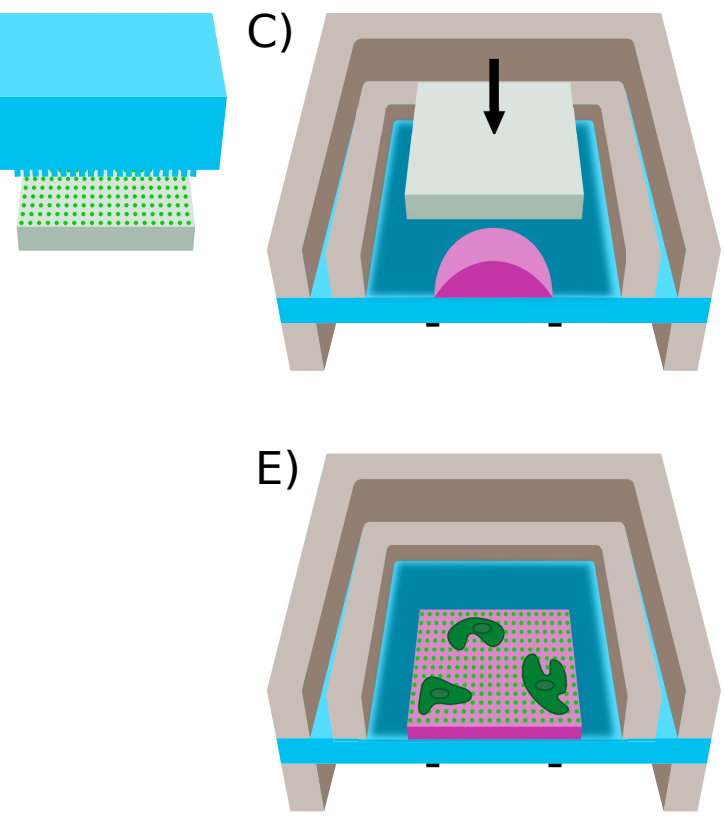

Figure 4. Process flow for the fabrication of a DEA-based device to stretch cells cultured on a thin layer of gel with an array of fluorescent dots. A) A DEA is fabricated by printing compliant electrodes on both sides of a prestretched silicone membrane. The top electrode is passivated with a thin layer of silicone elastomer, and a cell culture chamber is fixed on the membrane. B) An array of fluorescent fibronectin dots is patterned on a glass coverslip by micro-contact printing. C) The membrane is activated and a drop of polyacrylamide gel is placed at its surface, and then covered by the glass coverslip with the micropattern. D) The gel is left to crosslink for 1 hour, and then removed to expose a thin layer of gel covalently bonded to the silicone actuator and with a pattern of microdots at its surface. E) Cells are cultured on the gel, and the deformation of the array induced by the cell traction and the actuator strain is observed by fluorescence microscopy. 

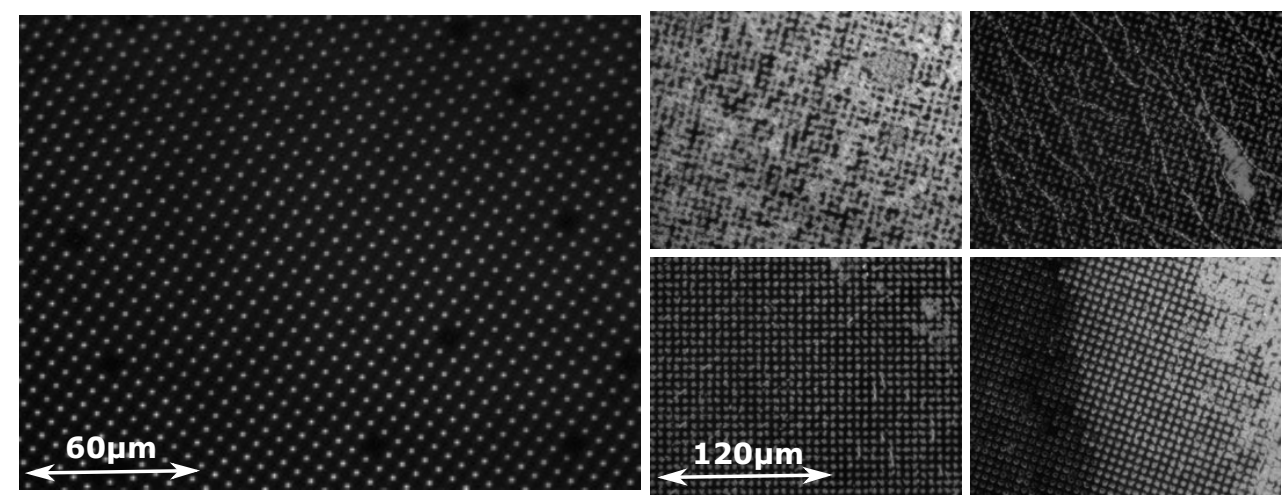

Figure 5. Left: portion of a good pattern of fluorescent fibronectin dots that can be used for experiments. Right: four examples of failed micro-patterning, leading to an array quality that is not good enough for experiments.

and the fibronectin is not transferred; too much force and the micropillars collapse, leading to a continuous layer of fibronectin. After stamping, the quality of the transfer is evaluated by observing the coverslips under a microscope with fluorescent illumination (Figure 5). The bad patterns can be discarded and the good patterns are kept to be transferred on the gel. The yield is quite low, and even good patterns are never good over the whole surface of the coverslip, hence the importance to maximize the zones on the actuator where the cells can be stretched and observed (the five $0.7 \mathrm{~mm} \times 4.5 \mathrm{~mm}$ in the current design) to increase the probability to have a portion of good-quality fluorescent dots where an experiment can take place.

Then, the surface of the actuator is activated to allow covalent bonding between the passivation layer and the gel that will be patterned on top. The activation of the surface is a sequence of 3 steps. 1) The surface is exposed to an oxygen plasma. We used a Diener Zepto plasma reactor at $50 \%$ of its power for 30 s. Exposure of DEAs to oxygen plasma is delicate, because it stiffens the surface of the elastomer membrane, and negatively affects the strain. ${ }^{8}$ However, the plasma step is crucial for the activation process, and too short an exposure leads to a failed activation and absence of adhesion of the gel on the silicone membrane. The parameters used here enable successful activation, while mitigating the stiffening effect. 2) The surface is exposed for 5 min to a $5 \%$ solution of (3-Aminopropyl)trimethoxysilane in ethanol, and then rinsed 3 times with DI water. 3) The surface is exposed for $30 \mathrm{~min}$ to a $0.5 \%$ solution of glutaraldehyde in water, and then rinsed 3 times with water.

The stiffness of the polyacrylamide gel solution is controlled by the ratio of acrylamide to bisacrylamide. To obtain a gel with a stiffness of $7.6 \mathrm{kPa}$, the following recipe is used. In a $15 \mathrm{ml}$ test tube, we combine $1250 \mu \mathrm{l}$ of an acrylamide solution ( $40 \%$ in water) with $350 \mu \mathrm{l}$ of a bisacrylamide solution ( $2 \%$ in water), $500 \mu l$ of a 10x PBS solution, and $2765 \mu \mathrm{l}$ of water. Then for every gel being made on an actuator, we transfer $969 \mu \mathrm{l}$ of the mixture into a $1.5 \mathrm{ml}$ test tube, to which we add $2 \mu \mathrm{l}$ of $\mathrm{N}, \mathrm{N}, \mathrm{N}^{\prime}, \mathrm{N}^{\prime}$-Tetramethylethylenediamine, $15 \mathrm{\mu l}$ of hydrochloric acid $1 \mathrm{~N}, 10 \mu \mathrm{l}$ of a solution of Acrylic acid N-hydroxysuccinimide ester ( $2 \mathrm{mg} \mathrm{ml}^{-1}$ in water), and finally $5 \mu \mathrm{l}$ of a solution of ammonium persulfate $\left(100 \mathrm{mg} \mathrm{ml}^{-1}\right.$ in water). As soon as we add the ammonium persulfate, the solution is placed on the activated surface of the actuator, and covered by a micro-patterned coverslip (Figure 4 C). The assembly is then placed in a desiccator connected to a vacuum pump and a nitrogen line. The desiccator is pumped and flushed with $\mathrm{N}_{2}$ several times. Then a small flow of Nitrogen is kept flowing in the desiccator for 30 minutes, to give time for the polyacrylamide gel to polymerise in the absence of oxygen. We then remove the coverslip, exposing a flat gel surface with a transferred array of micro-dots. We add cell culture media to the cell culture chamber and seed between 30 and 100 thousands cells (bovine aortic endothelial cells, or mouse embryonic fibroblasts). The cells require about 18 hours to attach to the gel and start exerting traction, after which an experiment can start.

\section{CHARACTERISATION OF THE ACTUATOR}

The strain of the actuator is measured using a USB camera, a Peta-pico-Voltron programmable high voltage power supply*, and a LabVIEW image processing routine tracking the corners of the culture zones (Figure 6).

\footnotetext{
*http://petapicovoltron.com
} 

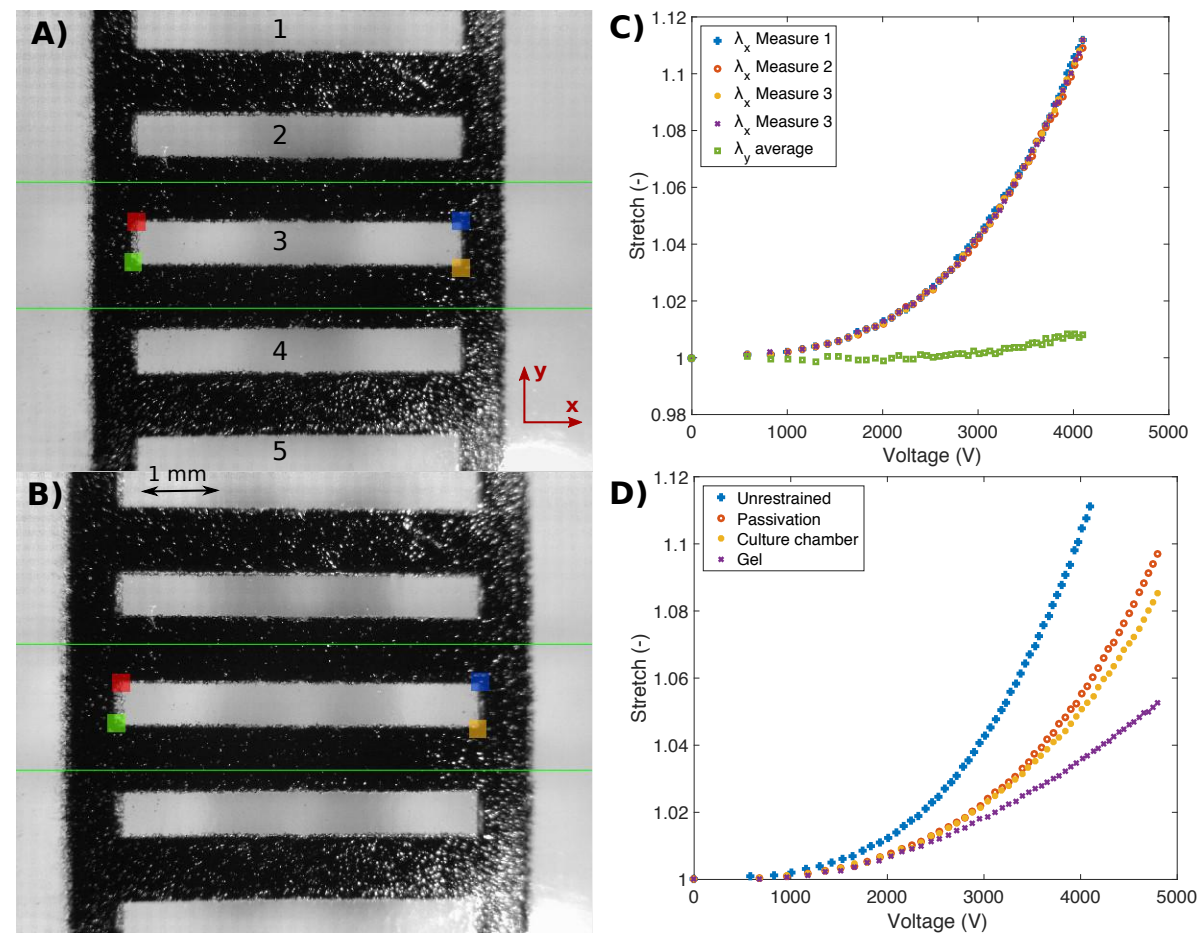

Figure 6. A) Actuator at rest with 5 zones (numbered 1 to 5 ) where cells can be stretched and observed. The corner of the cell culture zones are tracked to measure the strain. Only the zone between the two green lines (zone of interest) is analysed. Unless otherwise specified, the central zone (zone 3) is characterised. B) Actuator with 4500V applied, showing a horizontal stretch of 1.11. C) Stretch as a function of applied voltage in the two directions. The measurement shows excellent reproducibility on 4 measurements, as well as a good directional selectivity, as the strain in the perpendicular direction (y) is very low. D) Actuation stretch as a function of voltage at different steps of fabrication: Unrestrained (just after printing the two electrodes), passivation (after applying the passivation layer, corresponding to figure 3 left), Culture chamber (after placing the culture chamber, corresponding to figure 3 right), and gel (after making the polyacrylamide gel).

The repeatability is very good, as can be seen on figure $6 \mathrm{C}$ on an actuator before the passivation step. The directionality of the stretch is also excellent, with the strain in direction $x$ more than ten times higher than in direction $y$. The impact of the different fabrication steps on the strain is shown on figure $6 \mathrm{D}$. The unrestrained curve corresponds to the strain output just after printing the electrodes. Adding the passivation layer reduces the strain, because of the additional material added to the membrane. Placing the cell culture chamber also reduces the strain, because it decreases the amount of passive membrane around the electrodes, leading to a more important decrease of the tensile force in the membrane. ${ }^{9}$ Finally adding the gel causes an additional decrease of the strain to to the combined effect of the stiffening induced by the oxygen plasma, and the additional passive gel layer. For this actuator, the gel was thick (about $160 \mu \mathrm{m})$, while later gels were made thinner $(100 \mu \mathrm{m})$. But even with a thick gel, a strain larger than $5 \%$ is achieved, which is the required range for the stretching experiments envisaged here.

The geometry of the actuator, a rectangular electrode on a circular membrane, is not axisymmetric. In the direction of actuation $(x)$, the length of passive membrane separating the actuation zone from the clamped edge is different between zones. It is maximal for zone 3 (at the centre) and shorter for zone 5 . Because the amount of passive zone plays an important role in the amplitude of the actuation strain, ${ }^{9}$ it is important to verify the uniformity of the strain over the complete actuator. The actuation stretch is first measured using the corners of the actuation zone (the method used on figure 6), on the 5 different zones (Figure $7 \mathrm{~A}$ ). We name this the global stretch, and it can be seen that the two external zones exhibit a lower actuation stretch compared to the 3 zones which are closer to the centre. Then, we characterised the stretch at the centre of each of the 5 zones $(1 \mathrm{~mm} \times 0.7 \mathrm{~mm})$, using marks that were added to the electrode cliché as tracking points (local stretch). The 

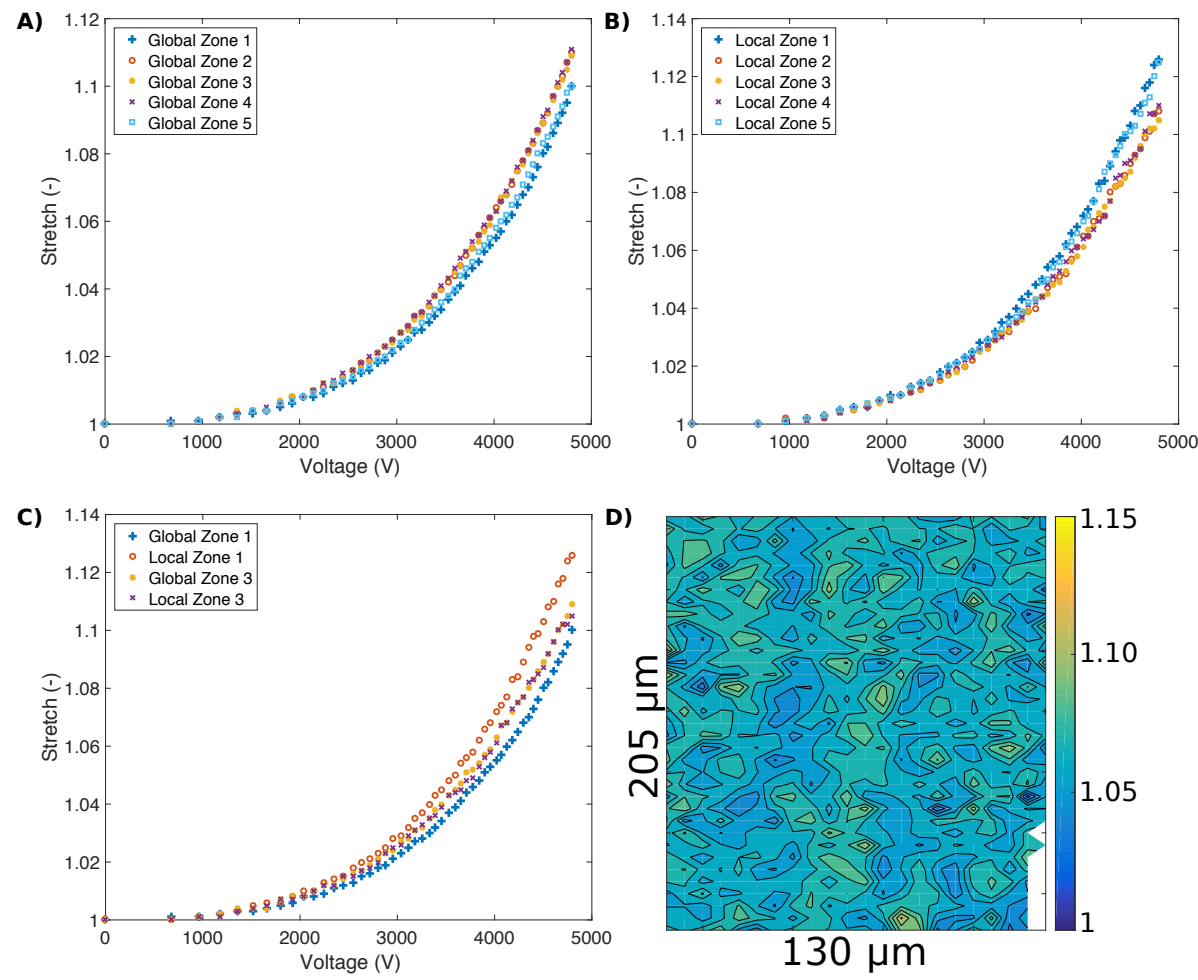

Figure 7. A) Global stretch as a function of voltage for the 5 different locations, when the measurement is taken on the complete stretching zone (method described on figure 6). B) Local stretch of the different location when measured at the centre of each zone. C) Comparison of the global and local stretch for the central zone and one of the external zones. D) Local stretch map computed from the position of the fluorescent dots.

results show that the two zones at the outside of the actuator exhibit more actuation than the central zones (Figure $7 \mathrm{~B}$ ), which is the opposite behaviour than what is observed for the global actuation. The comparison of the global/local stretch for the central zone (zone 3), and one of the external zones (zone 1) show that the global and local stretches are equal at the center of the actuator, whereas the external actuation zone shows more strain at the centre than globally, a sign of inhomogeneity in the stretch profile (Figure $7 \mathrm{~B}$ ). The fact that the strain is dependent of the location is not problematic, as it can be directly measured during the experiment by using the fluorescent dots. Figure $7 \mathrm{D}$ show a strain map obtained with a $\mathrm{x} 40$ objective using the patterned fluorescent dot for a voltage leading to an average stretch of $6 \%$.

Experiments are carried out on a zone that meet the following requirements:

- It must be located in one of the 5 transparent actuation zones defined by the electrodes. It is indeed the only location on the membrane that combines uniaxial tensile strain upon actuation, and transparency to allow for in-situ transmission microscopy.

- The chosen zone must have a good quality pattern of fluorescent microdots so that their position can be easily monitored by the image processing routines.

- The chosen zone must have a healthy cell (or cluster of a few cells depending on the experiment), well attached to the fibronectin dots, spread on the substrate and exerting traction.

Because of the low density at which cells are seeded in order to obtain single isolated cells (or clusters of a few cells) on the gel, it can be difficult to find a zone fulfilling the three conditions. If several good zones exist, the best choice is a zone as close as possible to the centre of the membrane. Indeed, away from the centre of the membrane, the stretch of a membrane element goes hand in hand with a translation of this element. Because of 


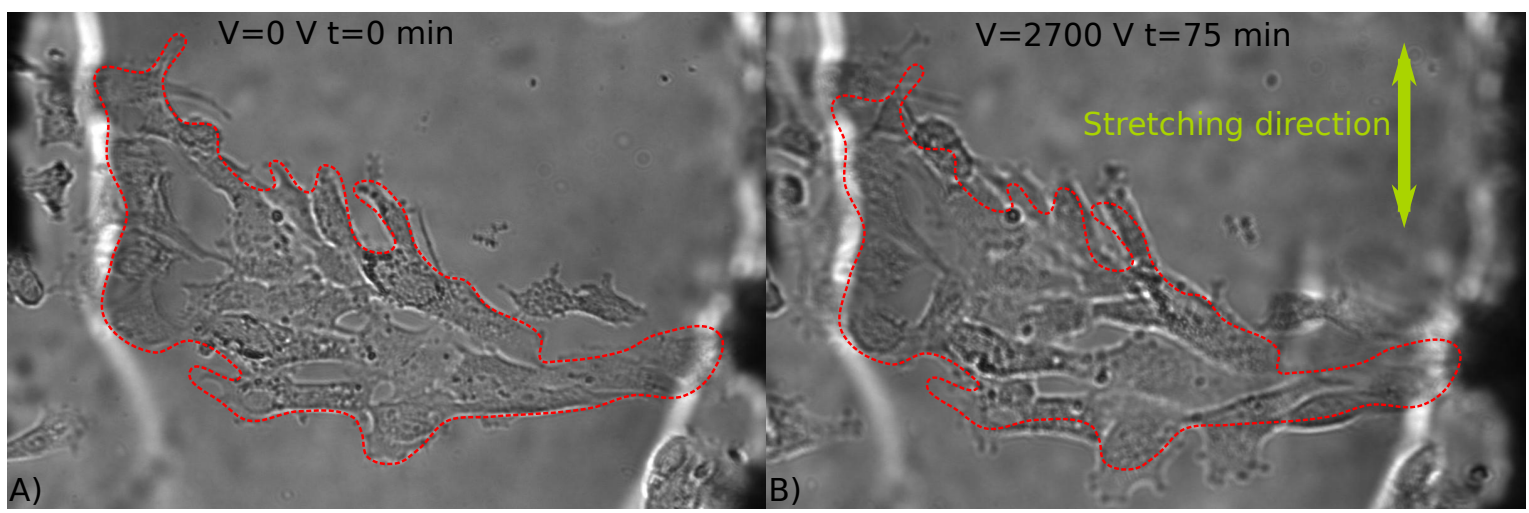

Figure 8. A) Initial picture of a bovine aortic endothelial cells cluster without applied voltage. B) After 75 min. at $2700 \mathrm{~V}$ (6\% strain along indicated direction). The outline of the unstrained cluster is indicated to highlight the strain.

the large magnification objectives used (typically $\mathrm{x} 40$ ), the observed field of view is small, and it is important that the observed cell remains visible while being stretched.

\section{INITIAL EXPERIMENTS WITH CELLS}

Preliminary experiments were made on cells to test that they attach and can be stretched. A cell viability test was performed by observing a cluster of cells attached on the actuator at rest and with a $6 \%$ strain applied (Figure 8). After 75 minutes of continuous strain, the cells remain attached and alive, and are unaffected by the high electric field applied to the actuator. This is, as was also shown in one of our previous study, ${ }^{3}$ because the actuator is designed to minimize the field present in the transparent zone where the cells are cultured.

We are interested to measure the change in cell traction forces when the cells are submitted to a rapid change of strain state, and we therefore want to submit the cells to a step of strain, from $0 \%$ to about $5 \%$. We tested the adhesion of cells on the matrix of dots by submitting them to different strain rate. We applied $5 \%$ strain (corresponding to $4200 \mathrm{~V}$ for the actuator used for the test) using a voltage ramp lasting $10 \mathrm{~s}, 5 \mathrm{~s}$, and about $250 \mu \mathrm{s}$ (the fastest switching speed of the Peta-pico-Voltron high-voltage power supply). The cells remained attached to the matrix of dots for all of the tested strain rates, showing that applying the strain rapidly is not a problem for the cells.

Experiments are currently under way to characterise the traction force of cells cultured on the actuator and study their response to applied strain.

\section{CONCLUSIONS}

Dielectric elastomer actuators are a promising actuation technology for biomedical application, and mechanotransduction studies in particular. They allow a compact design that makes it possible to conduct in-situ experiments directly under the microscope. The limited change of focal plane while stretching is also an important asset for direct observation under a microscope. We have shown how a soft polyacylamide gel can be covalently bonded to the surface of a silicone-based DEA, and can serve as a soft intermediary layer that cell can deform when they exert traction, but which can also be used to transfer strain to the cells. A fabrication methodology and basic cell survival studies have been presented, and experiments are currently underway to use the DEA-based stretcher to characterise the traction response of cells submitted to strain.

\section{ACKNOWLEDGMENTS}

The authors wish to thank Han Xu for the help with the cell culture and for providing cells; Joyce Wong and JoAnn Buczek-Thomas for access to their lab infrastructure; Matt Jacobsen, Shannon Anderson, and Anthony Maita for helpful discussion and general (but important) daily entertainment in the lab. 


\section{REFERENCES}

1. V. Vogel and M. Sheetz, "Local force and geometry sensing regulate cell functions," Nature Reviews Molecular Cell Biology 7, pp. 265-275, feb 2006.

2. E. P. Canovic, D. T. Seidl, S. R. Polio, A. A. Oberai, P. E. Barbone, D. Stamenovic, and M. L. Smith, "Biomechanical imaging of cell stiffness and prestress with subcellular resolution," Biomech Model Mechanobiol 13, pp. 665-678, Jun 2014.

3. A. Poulin, C. S. Demir, S. Rosset, T. Petrova, and H. R. Shea, "Dielectric elastomer actuator for mechanical loading of 2D cell cultures," Lab on a Chip 16(19), pp. 3788-3794, 2016.

4. S. Akbari, S. Rosset, and H. R. Shea, "Improved electromechanical behavior in castable dielectric elastomer actuators," Applied Physics Letters 102(7), p. 071906, 2013.

5. S. Akbari and H. R. Shea, "An array of 100um x 100um dielectric elastomer actuators with $80 \%$ strain for tissue engineering applications," Sensors and Actuators A: Physical 186, pp. 236 - 241, 2012.

6. S. Rosset, O. A. Araromi, S. Schlatter, and H. R. Shea, "Fabrication process of silicone-based dielectric elastomer actuators," Journal of Visualized Experiments 108, p. e53423, 2016.

7. A. Poulin, S. Rosset, and H. R. Shea, "Printing low-voltage dielectric elastomer actuators," Applied Physics Letters 107(24), p. 244104, 2015.

8. O. A. Araromi, S. Rosset, and H. R. Shea, "High-resolution, large-area fabrication of compliant electrodes via laser ablation for robust, stretchable dielectric elastomer actuators and sensors," ACS Applied Materials and Interfaces 7(32), pp. 18046-18053, 2015.

9. S. Rosset, O. A. Araromi, and H. R. Shea, "Maximizing the displacement of compact planar dielectric elastomer actuators," Extreme Mechanics Letters 3, pp. 72-81, 2015. 